\title{
Bronchial fistulae in ARDS patients: management with an extracorporeal
} lung assist device

\author{
M. Hommel*, M. Deja*, V. von Dossow*, K. Diemel" ${ }^{*}$ C. Heidenhain ", \\ C. Spies* and S. Weber-Carstens*
}

ABSTRACT: Patients with bronchial tree lesions feature, in particular, a high risk for developing bronchial fistulae after surgical repair when the clinical situation is complicated by acute lung injury (ALI)/acute respiratory distress syndrome (ARDS) and mechanical ventilation is needed. The current authors hypothesised that extracorporeal carbon dioxide removal would significantly decrease inspiratory airway pressures, thus promoting the protection of surgical bronchial reconstruction.

Four patients were studied after surgical reconstruction of bronchial fistulae in whom ALI/ARDS developed and mechanical ventilation with positive end-expiratory pressure was required. Gas exchange, tidal volumes, airway pressures, respiratory frequency, vasopressor and sedation requirements were analysed before and after initiation of a pumpless extracorporeal lung assist device ( $p E C L A ; N$ NovaLung ${ }_{\mathbb{R}}$, Talheim, Germany). Initiation of pECLA treatment enabled a reduction of inspiratory plateau airway pressures from 32.4 to $28.6 \mathrm{cmH}_{2} \mathrm{O}$ (3.2 to $2.8 \mathrm{kPa}$ ), effectively treated hypercapnia (from 73.6 to $53.4 \mathrm{mmHg}(9.8$ to $7.1 \mathrm{kPa}$ )) and abolished respiratory acidosis (from $\mathrm{pH} 7.24$ to 7.41 ). All patients survived and were discharged to rehabilitation clinics.

In patients after surgical bronchial reconstruction that was complicated by acute lung injury/ acute respiratory distress syndrome, use of pumpless extracorporeal carbon dioxide removal was safe and efficient. Initiation of a pumpless extracorporeal lung assist device enabled a less invasive ventilator management, which may have contributed to healing of surgical bronchial repair.

KEYWORDS: Acute respiratory distress syndrome, bronchial fistula, extracorporeal carbon dioxide removal, mechanical ventilation, pumpless extracorporeal lung assist device

$\mathbf{R}$ uptures or fistulae of the bronchial tree after pulmonary surgery, e.g. lobectomy, have been reported with an incidence of $1.5-28 \%$ [1]. Inflammatory diseases seem to be an important risk factor for the development of bronchial fistulae $(7.8 \%)$, especially in mechanically ventilated patients [1]. Positive pressure ventilation results in a considerable risk, in patients with bronchopleural fistula, of perpetuating the bronchial fistula and compromising surgical repair. Mortality has been reported as high as $67 \%$ in patients with bronchial fistulae receiving mechanical ventilation [1]. Mechanical ventilation applying positive airway pressures, therefore, should be avoided whenever possible, and early extubation is a major goal in perioperative therapy.

However the treatment of ruptures or fistulae of the bronchial tree remains a challenging surgical and intensive care medical problem. In the present case series, four patients were studied after surgical reconstruction of bronchial fistulae whose clinical course was complicated by acute lung injury/acute respiratory distress syndrome (ARDS) requiring high positive end-expiratory (PEEP) levels [2] and advanced ventilator support. It was hypothesised

\section{AFFILIATIONS}

*Dept of Anesthesiology and Intensive Care Medicine, Campus Virchow-Klinikum and Campus Mitte, "Dept of General, Visceral and Transplantation Surgery, Campus Virchow-Klinikum, Charité Universitätsmedizin Berlin, Berlin, and

\#Dept of Thoracic Surgery and Intensive Care Medicine, Hospital Großhansdorf, Großhansdorf, Germany.

\section{CORRESPONDENCE}

S. Weber-Carstens

Dept. of Anesthesiology and Intensive Care Medicine

Charité Universitätsmedizin Berlin

Campus Virchow-Klinikum and Campus Mitte

Augustenburger Platz 1

D-13353 Berlin

Germany

Fax: 4930450551909

E-mail: steffen.weber-carstens@

charite.de

Received:

February 112008

Accepted after revision:

August 042008

STATEMENT OF INTEREST

None declared. 
that extracorporeal carbon dioxide removal through a novel pumpless extracorporeal lung assist system (pECLA; Novalung ${ }_{\mathbb{R}}$ Talheim, Germany) would allow a reduction of inspiratory airway pressures, as shown by BEIN et al. [3], thereby promoting the protection of surgical bronchial reconstruction in these patients.

\section{CASE SERIES}

\section{Patient 1}

A 38-yr-old female sustained serious multiple trauma with a traumatic brain injury after falling out of a window. During damage-control surgery, the patient developed a significant fistula over the right-sided chest tube. The successive thoracotomy showed a tear of the right-middle lobe bronchus. After suture gas exchange deteriorated, in spite of differential lung ventilation, and the patient was transferred to the current authors' unit (ARDS referral centre of the Dept of Anesthesiology and Operative Intensive Care Medicine, Campus Virchow-Klinikum and Campus Mitte, Charité Universitätsmedizin Berlin, Berlin, Germany). Computed tomography at admission showed a lesion of the tracheal pars membranacea. Following a treatment algorithm designed by the current authors [4], pressure-controlled ventilation of both lungs with nitric oxide was established. The oxygenation improved markedly and an uneventful surgical repair of the tracheal rupture with a flap of the pectoralis muscle was performed within $24 \mathrm{~h}$. However, the patient developed nosocomial pneumonia and hypercapnia, and a consecutive increase of intracranial pressure occurred. It was decided that pECLA therapy should be initiated in order to maintain low tidal volume ventilation and concurrently control carbon dioxide tension. Lung function improved over the following 10 days and inhaled nitric oxide was discontinued on day 10; it was possible to remove the pECLA device on the following day. The patient was successfully weaned from mechanical ventilation on the 25th day of her intensive care unit (ICU) course and transferred back to the referring hospital two weeks later.

\section{Patient 2}

A 46-yr-old male received a resection of the oesophagus and a gastric pull-up for a carcinoma of the oesophagus. During the procedure, an injury to the left main stem bronchus had to be sutured. The patient had to be re-intubated 10 days after surgery and bronchoscopy showed a persisting lesion of the left main stem bronchus and the oesophagoscopy revealed an oesophageal-bronchial fistula. After stenting of the oesophagus, the patient developed progressive septic shock. Owing to the limited prognosis, the high operative risk and severe hypercapnia, pECLA therapy was initiated in order to control airway pressures and enable endobronchial stenting. A diagnostic workup for ongoing signs of inflammation showed a persisting oesophageal-bronchial fistula and resulted in resection of the gastric pull-up and a muscle flap reconstruction of the bronchus on day 21. The further course of the patient was complicated by persisting leakage of the main stem bronchus, despite further stenting attempts and differential lung ventilation. On day 26, bronchoscopy revealed a deepening of the bronchial lesion without local signs of inflammation, and a pneumectomy was performed. Subsequently, the patient recovered and was transferred for rehabilitation on day 50, when breathing spontaneously over the tracheostomy.

\section{Patient 3}

A 45-yr-old male received a sleeve resection for an adenocarcinoma of the left upper lobe. On post-operative day three the patient was transferred to the ICU for treatment of ARDS. Computed tomography on admission showed a bilobar pneumonia and a malperfusion of the left lower lobe artery. After initiation of inhaled nitric oxide and prone positioning, pulmonary function improved markedly. Owing to a persisting malperfusion of the left lower lobe, a pneumectomy was performed on day five. On day 19, the patient developed septic shock; computed tomography showed pneumonia and appropriate antimicrobial and supportive sepsis therapy was initiated. On day 20, a tension pneumothorax occurred. Bronchoscopy revealed an insufficiency of the stump and a muscle flap plastic was performed the following day. Postoperatively, the patient became progressively hypercapnic. Bronchoscopy on day 24 revealed a slight suture dehiscence and the decision to initiate pECLA therapy was made. Over the following days pulmonary function recovered and the patient developed no new leakage. The remaining suture dehiscence was successfully treated with a fibrin sealant 8 days later and pECLA-treatment discontinued the following day.

\section{Patient 4}

The 53 year old man receievd a pneumonectomy for bronchial carcinoma. Post-operatively he developed pneumonia of the remaining lung and impaired lung compliance led to high inspiratory airway pressures during mechanical ventilation. Bronchoscopy revealed a beginning stump insufficiency on day 6 and pECLA-therapy was initiated. Mechanical ventilation could be weaned progressively and pECLA-therapy could be weaned on day 15. The stump insufficiency was managed conservatively with drainage and could finally be sealed with fibrin on day 28 .

\section{Ventilatory management}

Table 1 outlines patient characteristics. All patients were managed according to the treatment algorithm used in the current authors' department for ARDS therapy, providing protective lung ventilation and including advanced therapeutic options, such as inhaled nitric oxide and prone positioning [4]. In all patients, the endotracheal tube could not be placed distal to the bronchial lesion and airway pressures affected the area of surgical repair. Prior to pECLA therapy, patients had been ventilated with tidal volumes of 4-6 mL per $\mathrm{kg}$ predicted body weight. PEEP values were adjusted to inspiratory oxygen fraction $\left(F \mathrm{I}, \mathrm{O}_{2}\right)$ following the low PEEP table according to BROWER et al. [5], as PEEP has been shown to influence the volume of bronchial fistulae in an experimental setting [6]. This resulted in median (interquartile range) PEEP values of 14 $(12.3-16.5) \mathrm{cmH}_{2} \mathrm{O}(1.37(1.20-1.61) \mathrm{kPa})$ and median inspiratory plateau pressures of 32.4 (29.8-35.9) $\mathrm{cmH}_{2} \mathrm{O}$ (3.17 (2.913.51) $\mathrm{kPa}$ ). Gas exchange was severely impaired, with median arterial oxygen tension $/ \mathrm{FI}, \mathrm{O}_{2}$ ratio of 141 (83-266) $\mathrm{mmHg}(18.8$ $(11.0-35.4) \mathrm{kPa}$ ) and a median arterial carbon dioxide tension of $73.6(67.1-78.5) \mathrm{mmHg}(9.79(8.92-10.4) \mathrm{kPa})$. Key measurement for initiation of pECLA treatment in the clinical setting was the combination of high inspiratory pressures and severe hypercapnia accompanied by respiratory acidosis [4]. 


\section{TABLE 1 Patient characteristics, treatment details and physiology assessment}

\begin{tabular}{|c|c|c|c|c|c|c|c|}
\hline Patient & Sex & Age yrs & ICU LOS days & Time on ventilator days & pECLA treatment days & APACHE-II score ${ }^{\#}$ & SOFA/SAPS-II score \\
\hline 1 & Female & 38 & 41 & 25 & 11 & 27 & $5 / 14$ \\
\hline 2 & Male & 46 & 58 & 40 & 7 & 21 & $15 / 79$ \\
\hline 3 & Male & 45 & 52 & 46 & 9 & 13 & $9 / 71$ \\
\hline 4 & Male & 53 & 36 & 22 & 9 & NA & NA \\
\hline
\end{tabular}

ICU: intensive care unit; LOS: length of stay; pECLA: pumpless extracorporeal lung assist; APACHE: acute physiology and chronic health evaluation; SOFA: sequential organ failure assessment; SAPS: simplified acute physiology score. *: on ICU admission; ": on pECLA initiation.

Table 2 shows that initiation of extracorporeal carbon dioxide removal allowed a reduction of median inspiratory airway pressures by further reducing tidal volume below $4 \mathrm{~mL}$ per $\mathrm{kg}$ predicted body weight. Control of hypercapnia was associated with a decreased respiratory rate and minute ventilation and allowed an increasing proportion of spontaneous breathing by weaning from sedation. Improving respiratory acidosis by extracorporeal carbon dioxide removal resulted in tapering of vasopressor therapy. Patients could be weaned from pECLA therapy after a median duration of 9 days with median (interquartile range) inspiratory plateau pressures of 25.5 (24.8-30.5) $\mathrm{cmH}_{2} \mathrm{O}(2.49(2.43-2.98) \mathrm{kPa})$ and a PEEP of 11 (9.8-12.0) $\mathrm{cmH}_{2} \mathrm{O}(1.08(0.96-1.17) \mathrm{kPa})$. On average, each patient needed two pECLA systems during the treatment period. No adverse effects of pECLA therapy were noted. Anticoagulation was achieved with continuous infusion of unfractionated heparin aiming at an activated partial thromboplastin time of 40-50 seconds. All four patients survived and were discharged to rehabilitation clinics.

\section{DISCUSSION}

The management of patients with acute respiratory failure after surgical repair of bronchial fistulae is challenging and reported cases are rare. In this situation, ventilatory support has to take into account two conflicting therapeutic goals: reducing airway pressures to protect bronchial reconstruction while maintaining sufficient oxygenation by application of an adequate ventilator therapy.

Implementation of pECLA was considered to represent a feasible and beneficial therapeutic tool in this situation for the following reasons: 1) inspiratory plateau airway pressures affecting the surgical repair of bronchial fistulae could be reduced; 2) as extracorporeal carbon dioxide removal reduces central respiratory drive [7], thereby improving patientventilator interaction, it facilitated the implementation of spontaneous breathing, supposedly reducing intrathoracic pressures; 3) extracorporeal carbon dioxide removal abolished respiratory acidosis with consecutive peripheral vasodilation and the need for vasoconstrictor treatment, such as norepinephrine. It is not possible to attribute the outcome of the patients to initiation of pECLA treatment on the basis of a case series of four patients, and randomised controlled trials are unlikely to be performed, owing to the limited number of patients in this clinical setting; however, from a pathophysiological point of view, all of the mentioned aspects had the potential to improve protection of the surgical bronchial reconstruction. Reduction of pulmonary shear stress may have directly protected surgical bronchial reconstruction [8, 9]. Spontaneous breathing as a part of a "lung protective ventilation strategy" by decreasing intrapulmonary shunt and intrathoracic pressures, as well as improving organ

\begin{tabular}{|c|c|c|c|c|}
\hline Parameter & Pre-pECLA & $12 \mathrm{~h}$ pECLA therapy & Day 2 pECLA therapy & Day 4 pECLA therapy \\
\hline Inspiratory plateau pressure $\mathrm{cmH}_{2} \mathrm{O}$ & $32.4(29.8-35.9)$ & $28.6(28.8-31.3)$ & $27.1(26.3-32.3)$ & $27.6(25.6-31.0)$ \\
\hline PEEP $\mathrm{cmH}_{2} \mathrm{O}$ & $14.0(12.3-16.5)$ & $15.0(12.8-16.5)$ & $15.5(12.8-16.8)$ & $14.5(10.3-15.8)$ \\
\hline Minute ventilation L. $\mathrm{min}^{-1}$ & $8.4(7.2-10.0)$ & $6.9(4.1-7.2)$ & $6.0(4.6-6.9)$ & $5.3(3.2-7.5)$ \\
\hline Assisted spontaneous breathing ${ }^{\#} \%$ & $7.2(0-23.1)$ & $17.4(0-38.3)$ & $27.7(2.9-58.2)$ & $40.7(7.4-71.9)$ \\
\hline $\mathrm{pH}$ & $7.24(7.10-7.37)$ & $7.41(7.23-7.46)$ & $7.37(7.32-7.43)$ & $7.38(7.29-7.43)$ \\
\hline $\mathrm{Pa}, \mathrm{CO}_{2} \mathrm{mmHg}$ & $73.6(67.1-78.5)$ & $53.4(37.4-59.8)$ & $50.3(49.1-57.8)$ & $54.0(53.3-65.3)$ \\
\hline $\mathrm{Pa}, \mathrm{O}_{2} / \mathrm{Fl}_{1} \mathrm{O}_{2} \mathrm{mmHg}$ & $141(83-266)$ & $166(137-232)$ & $147(119-224)$ & $201(116-315)$ \\
\hline Norepinephrine $\mu \mathrm{g} \cdot \mathrm{kg}^{-1} \cdot \mathrm{min}^{-1}$ & $0.47(0.12-0.76)$ & $0.30(0.08-0.48)$ & $0.18(0.06-0.22)$ & $0.09(0.07-0.19)$ \\
\hline
\end{tabular}

Data are presented as median (interquartile range). PEEP: positive end-expiratory pressure; PBW: predicted body weight; $\mathrm{Pa}, \mathrm{CO}_{2}$ : arterial carbon dioxide tension; $\mathrm{Pa}, \mathrm{O}_{2}$ : arterial oxygen tension; $\mathrm{Fl}_{1} \mathrm{O}_{2}$ : inspiratory oxygen fraction. ${ }^{*}$ : proportion of minute volume that was assisted spontaneous ventilation. $1 \mathrm{mmHg}=1.36 \mathrm{cmH} \mathrm{H}_{2} \mathrm{O}=0.133 \mathrm{kPa}$. 
perfusion $[10,11]$, might have been particularly beneficial in protecting surgical bronchial reconstruction. Furthermore, the reversal of respiratory acidosis and the succeeding decrease of vasopressor dosage might have supported wound healing [12]. Future clinical experiences have to show whether the aforementioned advantages in post-operative management in this clinical setting may potentially offset the substantial additional costs that can be attributed to pECLA treatment.

So far, there have been several case reports of extracorporal membrane oxygenation for the successful management of bronchopleural fistulae [13-15]. Unlike pECLA treatment, pumpdriven extracorporal membrane oxygenation (vvECMO) offers considerable quantity of oxygen transfer along with carbon dioxide removal, but with substantial documented risk [16] and, therefore, should be limited to severely hypoxaemic patients. The main advantage of the pumpless pECLA system compared with vvECMO therapy is diminished technical failure of the system and reduced bleeding complications, owing to a small extracorporeal surface area. However, the main complication of pECLA therapy remains ischaemia of the arterially cannulated leg. In patients without profoundly impaired oxygenation the less invasive interventional lung assist system ( $p E C L A ;$ Novalung ${ }^{\circledR}$ ) offers the advantage of sufficient carbon dioxide removal with potentially reduced risks of complications [3].

\section{Conclusion}

In patients who have previously undergone surgical bronchial reconstruction that was complicated by acute lung injury/ acute respiratory distress syndrome, the use of pumpless extracorporeal carbon dioxide removal was safe and efficient. Initiation of pumpless extracorporeal lung assistance contributed to less invasive ventilator management, which may have supported the healing of surgical bronchial repair.

\section{ACKNOWLEDGEMENTS}

The authors would like to acknowledge, in particular, $\mathrm{H}$. Weidemann for providing years of surgical expertise and backup, without which the successful treatment of these patients would not have been possible.

\section{REFERENCES}

1 Lois M, Noppen M. Bronchopleural fistulas: an overview of the problem with special focus on endoscopic management. Chest 2005; 128: 3955-3965.

2 Villar J, Kacmarek RM, Perez-Mendez L, Aguirre-Jaime A. A high positive end-expiratory pressure, low tidal volume ventilatory strategy improves outcome in persistent acute respiratory distress syndrome: a randomized, controlled trial. Crit Care Med 2006; 34: 1311-1318.

3 Bein T, Weber F, Philipp A, et al. A new pumpless extracorporeal interventional lung assist in critical hypoxemia/hypercapnia. Crit Care Med 2006; 34: 1372-1377.

4 Deja M, Hommel M, Weber-Carstens S, et al. Evidence-based therapy of severe acute respiratory distress syndrome: an algorithm-guided approach. J Int Med Res 2008; 36: 211-221.
5 Brower RG, Lanken PN, MacIntyre N, et al. Higher versus lower positive end-expiratory pressures in patients with the acute respiratory distress syndrome. N Engl J Med 2004; 351: 327-336.

6 Dennis JW, Eigen H, Ballantine TV, Grosfeld JL. The relationship between peak inspiratory pressure and positive end expiratory pressure on the volume of air lost through a bronchopleural fistula. J Pediatr Surg 1980; 15: 971-976.

7 Fiamma MN, Straus C, Thibault S, Wysocki M, Baconnier P, Similowski T. Effects of hypercapnia and hypocapnia on ventilatory variability and the chaotic dynamics of ventilatory flow in humans. Am J Physiol Regul Integr Comp Physiol 2007; 295: R1985-R1993.

8 Iglesias M, Jungebluth P, Petit C, et al. Extracorporeal lung membrane provides better lung protection than conventional treatment for severe postpneumonectomy noncardiogenic acute respiratory distress syndrome. I Thorac Cardiovasc Surg 2008; 135: 1362-1371.

9 Iglesias M, Martinez E, Badia JR, Macchiarini P. Extrapulmonary ventilation for unresponsive severe acute respiratory distress syndrome after pulmonary resection. Ann Thorac Surg 2008; 85: 237-244.

10 Putensen $\mathrm{C}$, Zech S, Wrigge $\mathrm{H}$, et al. Long-term effects of spontaneous breathing during ventilatory support in patients with acute lung injury. Am J Respir Crit Care Med 2001; 164: 43-49.

11 Putensen C, Mutz NJ, Putensen Himmer G, Zinserling J. Spontaneous breathing during ventilatory support improves ventilation-perfusion distributions in patients with acute respiratory distress syndrome. Am J Respir Crit Care Med 1999; 159: 1241-1248.

12 Saito T, Tazawa K, Yokoyama Y, Saito M. Surgical stress inhibits the growth of fibroblasts through the elevation of plasma catecholamine and cortisol concentrations. Surg Today 1997; 27: 627-631.

13 Khan NU, Al-Aloul M, Khasati N, Machaal A, Leonard CT, Yonan N. Extracorporeal membrane oxygenator as a bridge to successful surgical repair of bronchopleural fistula following bilateral sequential lung transplantation; a case report and review of literature. J Cardiothorac Surg 2007; 2: 28.

14 Rossaint R, Slama K, Lewandowski K, et al. Major thoracic surgery during long-term extracorporeal lung assist for treatment of severe adult respiratory distress syndrome (ARDS). Eur J Cardiothorac Surg 1992; 6: 43-45.

15 Dunser M, Hasibeder W, Rieger M, Mayr AJ. Successful therapy of severe pneumonia-associated ARDS after pneumonectomy with ECMO and steroids. Ann Thorac Surg 2004; 78: 335-337.

16 Morris AH, Wallace CJ, Menlove RL, et al. Randomized clinical trial of pressure-controlled inverse ratio ventilation and extracorporeal $\mathrm{CO}_{2}$ removal for adult respiratory distress syndrome. Am J Respir Crit Care Med 1994; 149: 295-305. 